\title{
Microbiological Quality of Some Puerto Rican Fast Foods. II. Retail and Consumer Level; Frozen or Fried ${ }^{1}$
}

\author{
F. Fernández-Coll ${ }^{2}$
}

\begin{abstract}
Samples of selected frozen Puerto Rican foods were purchased at the retail level and analyzed microbiologically. There was variation in the microbiological quality of these foods, particularly among brands within a given product. Some samples had high coliform and fecal coliform counts, although no coagulase positive staphylococci or Salmonella was found in any sample tested. Some of these foods were cooked according to processor's instructions and then held warm for up to $4 \mathrm{hr}$ at $38^{\circ} \mathrm{C}$ to reproduce conditions prevailing in routine practice in some cafeterias and fast food establishments. Results demonstrated that total bacterial counts were greatly diminished and in most cases completely eliminated, and that coliforms and fecal coliforms were completely destroyed in all cases by the cooking process. Holding foods warm for $4 \mathrm{hr}$ after cooking did not increase the microbial content of any of the samples. Provided post cooking contamination is avoided, holding these cooked foods at warm temperatures does not affect their microbiological quality.
\end{abstract}

\section{INTRODUCTION}

The current trend toward an ever increasing consumption of convenience and prepared foods has prompted studies regarding their microbial quality and safety. The wide variety of existing products does not make this determination an easy one.

Many of what are considered convenience foods belong to the frozen food category. Frozen foods are not sterilized; therefore, a potential of foodborne illness always exists. Although previous observations $(3,7)$ indicate that frozen foods do not appear to present a public health hazard, the presence of bacteria of public health significance has been demonstrated in commercially prepared frozen foods. Coagulase-positive strains of Staphylococcus aureus were isolated by Gunderson et al. (5) from precooked frozen foods. Saleh and Ordal (10) detected botulinum toxin in grossly-mishandled precooked chicken à la King. Salmonella spp. and coagulase-positive staphylococci were insolated by CanaleParola and Ordal (2) from commercially prepared precooked frozen poultry pies. Raj and Liston (9) reported that in fish homogenate, Staphylococcus aureus and Salmonella typhimurium survived through the fifth cycle of defrosting and freezing in sufficient numbers to constitute a public health hazard.

While it is true that heating these frozen products according to the

${ }^{1}$ Manuscript submitted to Editorial Board February 21, 1984.

${ }^{2}$ Assistant Food Microbiologist, Food Technology Laboratory, Agricultural Experiment Station, Mayagüez Campus, University of Puerto Rico, Rio Piedras, P.R. 
directions furnished by the processor will likely destroy the majority of the microorganisms present, sterility is not likely achieved. Illness from heat-stable toxins formed by staphylococci always remains a possibility. Moreover, evidence has been presented (2) indicating that baking frozen poultry pies according to the given directions does not always destroy the potential food poisoning microorganisms which may be present in the product.

It is common practice in many cafeterias and fast food restaurants to keep certain cooked foods warm for varying periods of time. Many of these cooked foods consist of prepared frozen foods. The effect this practice has on product microbial quality and safety is an important aspect to be considered. It is therefore our interest to determine not only the microbiological quality of frozen foods produced in Puerto Rico, but also to determine whether it is safe to eat some of these foods once they are cooked and held warm for a period of time.

\section{MATERIALS AND METHODS}

Frozen vegetable protein, cheese and pizza "empanadillas" (turnovers) as well as meat/vegetable protein, and crabmeat tacos were purchased at various local supermarkets. They were subjected to microbiological analysis to determine Aerobic Plate Count (APC), yeast and molds, total coliforms, fecal coliforms, coagulase-positive staphylococci and Salmonella spp. The methodology used in preparing the samples and performing the analyses were those stipulated by the Food and Drug Administration (1) and by the American Public Health Association (11). Modifications of procedures not incorporated into the standard methods were made to account for the recovery of freeze-injured cells $(4,6,8)$.

Four additional samples of each product were deep fried in corn oil at $177^{\circ} \mathrm{C}\left(350^{\circ} \mathrm{F}\right)$ until golden brown and placed in a light bulb-heated display glass box at $38^{\circ} \mathrm{C}\left(100^{\circ} \mathrm{F}\right)$. The same microbiological analyses mentioned above were performed with each sample immediately after frying and after 1, 2 and $4 \mathrm{hr}$ of holding warm at $38^{\circ} \mathrm{C}$ in the display box.

\section{RESULTS AND DISCUSSIONS}

Table 1 shows summarized results of the raw frozen products. These demonstrate that there is variation in the microbiological quality of the foods analyzed at the retail level. This is especially noticeable among brands within a given product, as indicated by the broad range of microbial counts encountered in some products. For a more uniform microbial quality in these products, microbiological guidelines should be established by the local regulatory agencies. These guidelines could be based on the lowest microbial counts found in each food product category. 
TABLE 1.-Average ${ }^{1}$ counts per gram of frozen raw products

\begin{tabular}{|c|c|c|c|c|c|c|c|}
\hline Product & Aerobic plate counts & Molds & Yeasts & Total coliforms & Fecal coliforms & $\begin{array}{l}\text { Coagulase- } \\
\text { positive } \\
\text { staphylococci }\end{array}$ & $\begin{array}{l}\text { Salmonella } \\
\text { spp. }\end{array}$ \\
\hline Meat-vegetable protein "empanadilla" & $\begin{array}{c}1.1 \times 10^{6} \\
\left(1.0 \times 10^{2} \text { to } 3.3 \times 10^{6}\right)^{2}\end{array}$ & $\begin{array}{c}<10 \\
\left(<10 \text { to } 2.0 \times 10^{1}\right)\end{array}$ & $\begin{array}{c}8.0 \times 10^{1} \\
\left(1.8 \times 10^{1} \text { to } 1.6 \times 10^{2}\right)\end{array}$ & $\begin{array}{c}8.4 \times 10^{2} \\
\left(<10 \text { to } 3.0 \times 10^{3}\right)\end{array}$ & $\begin{array}{c}5.0 \times 10^{2} \\
\left(<10 \text { to } 1.8 \times 10^{3}\right)\end{array}$ & $<10$ & Neg \\
\hline Cheese "empanadilla" & $\begin{array}{c}7.5 \times 10^{4} \\
\left(2.1 \times 16^{4} \text { to } 2.2 \times 10^{5}\right)\end{array}$ & $\begin{array}{c}<10 \\
\left(<10 \text { to } 2.0 \times 10^{1}\right)\end{array}$ & $\begin{array}{c}2.2 \times 10^{2} \\
\left(5.0 \times 10^{1} \text { to } 7.0 \times 10^{2}\right)\end{array}$ & $\begin{array}{c}2.3 \times 10^{2} \\
\left(<10 \text { to } 5.8 \times 10^{2}\right)\end{array}$ & $\begin{array}{c}7.6 \times 10^{1} \\
\left(<10 \text { to } 1.6 \times 10^{2}\right)\end{array}$ & $<10$ & Neg \\
\hline Pizza "empanadilla" & $\begin{array}{c}2.1 \times 10^{6} \\
\left(3.6 \times 10^{4} \text { to } 6.5 \times 10^{6}\right)\end{array}$ & $\begin{array}{c}<10 \\
\left(<10 \text { to } 5.0 \times 10^{1}\right)\end{array}$ & $\begin{array}{c}4.2 \times 10^{2} \\
\left(3.0 \times 10^{1} \text { to } 2.6 \times 10^{3}\right)\end{array}$ & $\begin{array}{c}2.4 \times 10^{2} \\
\left(<10 \text { to } 8.4 \times 10^{2}\right)\end{array}$ & $\begin{array}{c}1.3 \times 10^{2} \\
\left(<10 \text { to } 4.6 \times 10^{2}\right)\end{array}$ & $<10$ & Neg \\
\hline Meat-vegetable protein tacos & $\begin{array}{c}1.2 \times 10^{5} \\
\left(1.9 \times 10^{3} \text { to } 2.4 \times 10^{5}\right)\end{array}$ & $\begin{array}{c}<10 \\
\left(<10 \text { to } 1.0 \times 10^{1}\right)\end{array}$ & $\begin{array}{c}1.4 \times 10^{3} \\
\left(3.0 \times 10^{1} \text { to } 2.7 \times 10^{3}\right)\end{array}$ & $\begin{array}{c}1.5 \times 10^{4} \\
\left(<10 \text { to } 3.0 \times 10^{4}\right)\end{array}$ & $\begin{array}{c}7.5 \times 10^{3} \\
\left(<10 \text { to } 1.5 \times 10^{4}\right)\end{array}$ & $<10$ & Neg \\
\hline Crabmeat tacos & $3.0 \times 10^{4}$ & $<10$ & $<10$ & $<10$ & $<10$ & $<10$ & Neg \\
\hline
\end{tabular}

${ }^{1}$ Based on 6, 5, 7, 2 and 1 different sets of samples of meat-vegetable protein "empanadilla", cheese "empanadilla", pizza flavored "empanadilla", meat-vegetable protein tacos and crabmeat tacos, respectively.

${ }^{2}$ Numbers in parentheses indicate range of microbial counts encountered. 
TABLE 2.-Average $e^{1}$ counts per gram of fried products immediately after cooking and at varied periods of time of holding warm at $38^{\circ} \mathrm{C}$

\begin{tabular}{|c|c|c|c|c|c|c|c|}
\hline Product & $\begin{array}{l}\text { Aerobic plate } \\
\text { counts }\end{array}$ & Molds & Yeasts & $\begin{array}{l}\text { Total } \\
\text { coliforms }\end{array}$ & $\begin{array}{l}\text { Fecal } \\
\text { coliforms }\end{array}$ & $\begin{array}{l}\text { Coagulase } \\
\text { positive } \\
\text { staphylococci }\end{array}$ & $\begin{array}{l}\text { Salmonella } \\
\text { spp. }\end{array}$ \\
\hline \multicolumn{8}{|l|}{$\begin{array}{l}\text { Meat-vegetable protein "em- } \\
\text { panadilla" }\end{array}$} \\
\hline Immediately after frying & $<10$ & $<10$ & $<10$ & $<10$ & $<10$ & $<10$ & Neg \\
\hline After $1 \mathrm{hr}$ at $38^{\circ} \mathrm{C}$ & $\begin{array}{c}1.7 \times 10^{1}(<10 \text { to } \\
\left.8.0 \times 10^{1}\right)^{2}\end{array}$ & $<10$ & $<10$ & $<10$ & $<10$ & $<10$ & Neg \\
\hline After $2 \mathrm{hrs}$ at $38^{\circ} \mathrm{C}$ & $<10$ & $<10$ & $<10$ & $<10$ & $<10$ & $<10$ & Neg \\
\hline After 4. hrs at $38^{\circ} \mathrm{C}$ & $\begin{aligned}<10 & (<10 \text { to } 1.0 \\
& \left.\times 10^{1}\right)\end{aligned}$ & $<10$ & $<10$ & $<10$ & $<10$ & $<10$ & $\mathrm{Neg}$ \\
\hline \multicolumn{8}{|l|}{ Cheese "empanadilla" } \\
\hline Immediately after frying & $\begin{array}{c}1.0 \times 10^{1}(<10 \text { to } \\
\left.3.5 \times 10^{1}\right)\end{array}$ & $<10$ & $<10$ & $<10$ & $<10$ & $<10$ & Neg \\
\hline After $1 \mathrm{hr}$ at $38^{\circ} \mathrm{C}$ & $\begin{array}{l}3.0 \times 10^{1}(1.0 \times \\
10^{1} \text { to } 5.0 \times \\
\left.10^{1}\right)\end{array}$ & $<10$ & $<10$ & $<10$ & $<10$ & $<10$ & Neg \\
\hline After $2 \mathrm{hrs}$ at $38^{\circ} \mathrm{C}$ & $\begin{array}{c}2.0 \times 10^{1}(<10 \text { to } \\
\left.4.0 \times 10^{1}\right)\end{array}$ & $<10$ & $<10$ & $<10$ & $<10$ & $<10$ & Neg \\
\hline After 4 hrs at $38^{\circ} \mathrm{C}$ & $\begin{array}{c}3.0 \times 10^{1}(<10 \text { to } \\
\left.4.0 \times 10^{1}\right)\end{array}$ & $<10$ & $<10$ & $<10$ & $<10$ & $<10$ & Neg \\
\hline \multicolumn{8}{|l|}{ Pizza flavored "empanadilla" } \\
\hline Immediately after frying & $\begin{array}{c}7.5 \times 10^{1}(<10 \text { to } \\
\left.1.5 \times 10^{2}\right)\end{array}$ & $<10$ & $<10$ & $<10$ & $<10$ & $<10$ & Neg \\
\hline After $1 \mathrm{hr}$ at $38^{\circ} \mathrm{C}$ & $\begin{array}{c}8.5 \times 10^{1}(<10 \text { to } \\
\left.1.7 \times 10^{2}\right)\end{array}$ & $<10$ & $<10$ & $<10$ & $<10$ & $<10$ & Neg \\
\hline After $2 \mathrm{hrs}$ at $38^{\circ} \mathrm{C}$ & $\begin{aligned}<10 & (<10 \text { to } 1.0 \\
& \left.\times 10^{1}\right)\end{aligned}$ & $<10$ & $<10$ & $<10$ & $<10$ & $<10$ & Neg \\
\hline After 4 hrs at $38^{\circ} \mathrm{C}$ & $\begin{array}{c}1.0 \times 10^{1}(<10 \text { to } \\
\left.2.0 \times 10^{1}\right)\end{array}$ & $<10$ & $<10$ & $<10$ & $<10$ & $<10$ & Neg \\
\hline
\end{tabular}


Meat-vegetable protein tacos

Immediately after frying

\begin{tabular}{|c|c|c|c|c|c|c|}
\hline $\begin{array}{l}1.0 \times 10^{1}(1.0 \times \\
10^{1} \text { to } 3.0 \times \\
\left.10^{1}\right)\end{array}$ & $<10$ & $<10$ & $<10$ & $<10$ & $<10$ & Neg \\
\hline $\begin{array}{c}1.0 \times 10^{1}(<10 \text { to } \\
\left.7.0 \times 10^{1}\right)\end{array}$ & $<10$ & $<10$ & $<10$ & $<10$ & $<10$ & Neg \\
\hline $\begin{array}{c}2.0 \times 10^{1}(<10 \text { to } \\
\left.4.0 \times 10^{1}\right)\end{array}$ & $<10$ & $<10$ & $<10$ & $<10$ & $<10$ & $\mathrm{Neg}$ \\
\hline$<10$ & $<10$ & $<10$ & $<10$ & $<10$ & $<10$ & $\mathrm{Neg}$ \\
\hline $\begin{array}{c}1.0 \times 10^{1}(<10 \text { to } \\
\left.2.0 \times 10^{1}\right)\end{array}$ & $<10$ & $<10$ & $<10$ & $<10$ & $<10$ & $\mathrm{Neg}$ \\
\hline$<10$ & $<10$ & $<10$ & $<10$ & $<10$ & $<10$ & Neg \\
\hline $\begin{array}{l}2.0 \times 10^{1}(1.0 \times \\
10^{1} \text { to } 4.0 \times \\
\left.10^{1}\right)\end{array}$ & $<10$ & $<10$ & $<10$ & $<10$ & $<10$ & Neg \\
\hline$<10$ & $<10$ & $<10$ & $<10$ & $<10$ & $<10$ & Neg \\
\hline
\end{tabular}

○

After $1 \mathrm{hr}$ at $38^{\circ} \mathrm{C}$

After 2 hrs at $38^{\circ} \mathrm{C}$

After 4 hrs at $38^{\circ} \mathrm{C}$

Crabmeat tacos

Immediately after frying

After 1 hr at $38^{\circ} \mathrm{C}$

After 2 hrs at $38^{\circ} \mathrm{C}$

After 4 hrs at $38^{\circ} \mathrm{C}$

Based on 6, 5, 7, 2 and 1 different sets of samples for meat-vegetable protein empanadillas, cheese empanadillas, pizza flavored empanadillas, meat-vegetable protein tacos and crabmeat tacos respectively.

${ }^{2}$ Numbers in parentheses indicate range of microbial counts encountered. 
The amount of coliforms and fecal coliforms is high in certain products, thus indicating that greater care should be taken in plant sanitation and in possible temperature abuse during distribution and retail. However, no Salmonella nor coagulase-positive staphylococci were found in any of the samples tested.

Table 2 summarizes results obtained in the microbiological analysis of the cooked products, both immediately after frying and after various periods of holding warm at $38^{\circ} \mathrm{C}$ in a display box. These demonstrate that frying products according to processor's instructions almost eliminates, and completely in many instances, the microbial load present. Molds, yeasts, coliforms and fecal coliforms are completely destroyed by the cooking process. Keeping the fried products warm at $38^{\circ} \mathrm{C}$ for up to $4 \mathrm{hr}$ does not alter their microbiological quality. Therefore, provided there is no post cooking contamination, holding these cooked foods at warm temperatures does not adversely affect their microbiological quality.

\section{RESUMEN}

Debido a que los alimentos preservados a bajas temperaturas no son sometidos a un proceso de esterilización, existe el potencial de enfermedades alimentarias como consecuencia de su consumo. Se ha demostrado la presencia significativa de microorganismos desde el punto de vista de la salud pública en alimentos congelados.

Es una práctica común en muchas cafeterías y otros establecimientos que expenden alimentos preparados el mantener ciertos alimentos cocidos tibios por un período de tiempo que puede variar. El efecto que esta práctica tiene sobre la calidad microbiológica y la seguridad del alimento es un aspecto importante que debe considerarse. El interés del presente estudio es determinar la calidad microbiológica de alimentos congelados producidos en Puerto Rico y la seguridad de algunos de estos productos una vez se cocinan y luego se mantienen tibios durante un tiempo.

Se compraron en distintos supermercados muestras de empanádillas congeladas de carne con proteína vegetal, queso y pizza, así como tacos de carne con proteina vegetal y carne de jueyes. Algunas muestras se analizaron crudas mientras que otras se frieron en aceite de maíz a $177^{\circ}$ $\mathrm{C}\left(350^{\circ} \mathrm{F}\right)$ y luego se mantuvieron a $38^{\circ} \mathrm{C}\left(100^{\circ} \mathrm{F}\right)$ en una cámara por 1,2 y 4 hr luego de fritas.

Los resultados de las muestras crudas demostraron que existe una gran variabilidad entre estos productos, especialmente entre diferentes marcas del mismo producto, lo que señala la necesidad de establecer guias microbiológicas para asegurar uniformidad en estos productos. Algunas muestras arrojaban elevados números de coliformes y de coliformes 
fecales, mientras que otras no. No se encontró Salmonella ni estafilococos coagulasa-positivos en minguna de las muestras estudiadas.

Los resultados de los productos cocinados y mantenidos tibios demuestran que el cocinar elimina la mayoría, y en muchos casos, todos los organismos. Siempre y cuando no haya contaminación luego de freir, el mantener estos alimentos cocidos y tibios no afecta adversamente su calidad microbiológica.

\section{LITERATURE CITED}

1. Bacteriological Analytical Manual for Foods, 1976. U.S. Food and Drug Administration, Washington, D.C.

2. Canale-Parola, E. and Ordal, Z. J., 1957. A survey of the bacteriological quality of frozen poultry pies, Food Technol, 11: 578-82.

3. Dack, G. M., Wheaton, E., Mickelson, M. N. and Schuler, M. N., 1960. Public health significance of microorganisms in frozen pot pies, Quick Frozen Foods 22: 44-5; 160 62.

4. Flowers, R. S. and Ordal, Z. J., 1979. Current methods to detect stressed Staphylococci, J. Food Prot. 42: 362-67.

5. Gunderson, M. F., MacFadden, H. W. and Kyle, T. S., 1954. The Bacteriology of Commercial Poultry Processing, Burgess Publ. Co., Minneapolis, Minn.

6. Hartman, P. A., 1979. Modification of conventional methods for recovery of injured coliforms and Salmonellae, J. Food Prot. 42: 356-61.

7. Licciardello, J. J. and Hill, W. S., 1978. Microbiological quality of commercial frozen beef, poultry and tuna pies, J. Milk Food Technol. 20: 216-19.

8. Powers, E. M. and Lutt, T. G., 1979. Rapid enumeration and identification of stressed fecal coliforms, J. Food Prot. 42: 342-45.

9. Raj, H. and Liston, J., 1961. Survival of bacteria of public health significance in frozen seafoods, Food Technol. 15: 429-34.

10. Saleh, M. A. and Ordal, Z. J., 1955. Studies on growth and toxin production of Clostridium botulinum in a pre-cooked frozen food. I. Some factors affecting growth and toxin production, Food Research 20: 332.

11. Standard Methods for the Examination of Dairy Products, 1972. 13th ed, William J. Housler, Ed, American Public Health Association, Washington, D.C. 